\title{
Supporting Caring among Intergenerational Family Members through Family Fitness Tracking
}

\author{
Qingyang Li \\ Department of Informatics \\ University of California, Irvine \\ Irvine, CA, United States \\ qingyl6@uci.edu \\ Daniel A. Epstein \\ Department of Informatics \\ University of California, Irvine \\ Irvine, CA, United States \\ epstein@ics.uci.edu
}

\author{
Clara Caldeira \\ Department of Informatics \\ University of California, Irvine \\ Irvine, CA, United States \\ claram@uci.edu \\ Yunan Chen \\ Department of Informatics \\ University of California, Irvine \\ Irvine, CA, United States \\ yunanc@ics.uci.edu
}

\begin{abstract}
We present results from a qualitative study involving eight intergenerational families (27 participants) to understand how a family tracking intervention can help support care among intergenerational family members. Our findings show that family members communicate and stay aware of each other's' health through shared fitness data and conversations triggered by fitness sharing. We identified different challenges and preferences among the three age groups in our study: older adults enjoyed family fitness sharing but often encountered various technical challenges, the middle-aged group served as a key person to care for the rest of the family members, and the young generation could not fully engage in fitness sharing due to their busy schedule and privacy concerns. These findings suggest the design of family fitness sharing to account for the age differences in intergenerational families and support the unique needs of family fitness sharing.
\end{abstract}

\section{CCS CONCEPTS}

Human-centered computing $\rightarrow$ Human computer interaction (HCI) $\rightarrow$ HCI design and evaluation methods $\rightarrow$ Field studies

\section{KEYWORDS}

Intergenerational; families; care; personal informatics; fitness

\section{ACM Reference format:}

Qingyang Li, Clara Caldeira, Daniel A. Epstein, and Yunan Chen. 2020.

Supporting Caring among Intergenerational Family Members through

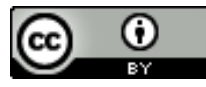

This work is licensed under a Creative Commons Attribution International 4.0 License. PervasiveHealth '20, May 18-20, 2020, Atlanta, GA, USA

2020 Copyright is held by the owner/author(s).

ACM ISBN 978-1-4503-7532-0/20/05.

https://doi.org/10.1145/3421937.3422018
Family Fitness Tracking. In PervasiveHealth'20: 14th EAI International Conference on Pervasive Computing Technologies for Healthcare (PervasiveHealth'20), May 18-20, 2020, Atlanta, GA, USA. ACM, Atlanta, GA, USA, 10 pages. https://doi.org/10.1145/3421937.3422018

\section{INTRODUCTION}

Family members have an inherent desire to communicate and stay aware of each other's health and well-being [15, 23, 25]. An increasing number of studies have focused on developing technology to promote family engagement in health and well-being $[3,7,9]$. However, sharing health information among family members could be challenging in an intergenerational family context [1]. Between parents and their adult children, lifestyle differences or living in different locations may result in families not communicating sufficiently. Different generations may also prefer different information channels, and older adults are often left behind since they may not on the same technology platform as their middle-aged children or younger generations.

Previous research on family health communication has discussed eating habits [21, 27], sleep qualities [24], familyfocused exercise games [26], and care-related behaviors [28]. These studies suggested that tracking in a family context could increase social support and help families build awareness, reflect on health behaviors, and set goals. Self-tracking tools with data sharing features have the opportunity to facilitate support, awareness, and advice about health conditions between older adults and their adult children [2]. However, it is unclear if self-tracking tools can initiate health-related conversations among intergenerational family members or how family members can implement these tools to support caring. In this paper, we explore how a family tracking intervention can help support care among intergenerational family members. Our study answers the following questions:

RQ1: How does sharing daily step counts influence health awareness of intergenerational family members? 
RQ2: How do family members express care through conversations triggered by fitness sharing?

RQ3: What are the challenges family members face when tracking and sharing fitness data?

To investigate these questions, we conducted a study using WeRun, a widely used fitness app, from July 2018 to April 2019. Different from many fitness tracking applications which operate as standalone apps, WeRun is embedded in WeChat, a messaging and social media app widely used in China. WeRun imports fitness data from compatible fitness tracking tools (e.g., Apple Health, Google Fit) and automatically shares real-time fitness data with all contacts of a WeChat account who have signed up with WeRun.

To understand how a family tracking intervention can support care in intergenerational families, we recruited eight family units (27 people in total) to use WeRun for two weeks. Participants used the number of steps taken by them and their families as a proxy to quantify their daily activities and measure health conditions of both themselves and their families. Family tracking triggered different levels of information sharing and varied forms of caring. The three generations displayed distinctly different use behaviors and experiences. Some older adult participants had difficulties using the technology, but they appreciated being able to better communicate with their children once they had mastered it. The middle-aged group often took a lead role in caring for the other generations, paying attention other family members' data to notice any daily differences. The younger generation focused on preserving privacy and engaged with the system minimally. Our results suggest that family tracking technologies can better respond to the needs of different generations.

Our study contributes to the HCI community by exploring the potential of family tracking to support family care and extends existing knowledge on fitness tracking in a family context. Finally, we provide recommendations for the design of tracking tools that support caring among intergenerational families and account for the varied use patterns and preferences of different generations.

\section{RELATED WORK}

\subsection{Health Information Sharing in Family}

Technologies have been increasingly designed to support families' needs for awareness and communication around health, often supporting awareness of health status. Technology has explored how families could display what and how they are currently doing [22], share daily experiences and information [1, 14], and collaboratively achieve health behavior change goals [3, 7].

Although family members have the intrinsic desire to stay aware of one another's health and well-being [15, 23, 25], they may face challenges when sharing health-related information. For example, it can be challenging to foster positive sharing behaviors if the information shared appears open to criticism [1]. Instead, family members are more willing to share if feel they will receive care and positive feedback [17]. Generational challenges also emerge, as parents struggle to balance prying for information and showing care, while adult children struggle between staying independent and receiving care [1].

\subsection{Tracking in a Family Context}

Personal informatics literature examines the idea of tracking for self-understanding [18]. While most personal informatics research has focused on self-tracking, research is increasingly being conducted in a family context.

Substantial work has examined how people share physical activity data socially $[4,6]$. Particularly relevant to our work, Gui et al. [11] studied WeRun to understand how people shared fitness data in pre-existing social networks and found that participants did not pay much attention to the accuracy of fitness tracking data provided by WeRun. For most of their participants, competition was not a strong motivation to track fitness data. They were aware that their contacts had diverse ages, occupations, health statuses, and lifestyles, and considered a person's circumstances when interpreting and comparing their step counts.

In a family context, researchers studied how families track eating habits [21, 27], sleep [24], physical exercise [26], and carerelated behaviors [28]. These studies show that tracking in a family context has the opportunity to increase social support $[21,28]$ help families build awareness $[10,27]$, reflect on each other's health behaviors, and collaboratively achieve goals [24].

Similarly, Kaziunas et al.'s study introduces the concept of caring-through-data, where data is means of fostering empathy and togetherness, rather than exclusively used to help combat or address disease [17]. Some parents in their study wore a continuous glucose monitor in solidarity with their T1D children to promote empathy, relational intimacy, and compassion. They suggest that the meaning of both care and data can vary depending on the family, their social contexts, and their life stages.

Among these studies, participants also expressed privacy concerns around sharing their data with other family members. Yamashita et al. [29] found that sharing patients' private information with family caregivers might cause negative feelings of surveillance and violation of trust.

\subsection{Self-Tracking and Older Adults}

Having older adults leave their home to move into a retirement community can limit their autonomy and increase feelings of disenfranchisement and socio-cognitive limitations by providing unnecessary assistance [2]. "Aging in place" research aims to empower older adults to maintain independence in their residences. Successful aging in place relies on self-care activities for health management including regular self-tracking, such as blood pressure [2]. Compared with other age groups, older adults are more likely to use paper or medical devices to self-track, and less likely to use computers and mobile tools. For older adults, the most common 
Supporting Caring among Intergenerational Family Members by Family Fitness Tracking

self-tracking goal is to identify abnormalities in their health conditions [2]. Caldeira et al. [2] found that self-tracking tools which have functionality for sharing data within individuals' social networks can assist in communication around health conditions between older adults and their adult children.

\section{METHODS}

We conducted a study from July of 2018 to April of 2019 to understand how sharing fitness tracking data with WeRun might influence the health communication and care practices among older adults and their family members. Our study involved interviews and analysis of chatlog content.

\subsection{Participants}

We used snowball sampling to recruit participants through our social networks. We posted the study flyer on our WeChat personal homepages, called "Moment", asking friends to participate or to refer people to us. We then asked interested participants to invite their family members to participate. One of the authors was friends, coworkers, or acquaintances with one member of most of the recruited families, but did not know the rest of their family members. All participants used Mandarin to communicate as a family, and were located in the urban areas of central and eastern China throughout the study.

We divided participants into three age groups based on their working status. In China, the legal retirement age is 60 years old, so we therefore defined the older adults in this study were above 60 years old and had retired [8]. We defined middle-aged participants were those above 40 years old and were still working, and young participants were typically in their 20 or 30 's, either working professionals or students.

We recruited eight family units (27 people in total). Each family unit consisted of at least three adults (18 or above). We required all groups include at least one older adult and one from a younger generation. Among the 27 participants, 11 were older adults, 12 were middle-aged, and four were young participants. The older adults who participated in the study were autonomous, either generally healthy or having slight functional impairments. Table 3.1 shows the demographic information of the participants. We obtained IRB approval prior to recruitment.

\subsection{Study Design}

We invited our participants to join tracking-themed family chat groups and asked them to share their daily step counts collected by WeRun in those groups. We required families to participate in the study for at least two weeks. Families could then choose to either dissolve the family online group or continually engage. With their permission, we recorded their group chatlogs during the study until conversation ended. We also collected their daily step counts for 30 days prior to study and after the study began.
PervasiveHealth 20, May 18-20, 2020

Atlanta, United States, USA

Participants also had access to other social functions in WeRun. WeRun Ranking automatically ranks the daily steps of users and their contacts who also use WeRun. Users can "like" each other's step counts. Users can "follow" a specific contact, which places the contact's step count at the top of their WeRun page. WeRun sends a summary of users' daily step counts as a form of leaderboard at 10 pm every day.

Table 1: Demographics of participants

\begin{tabular}{|c|c|c|c|c|c|c|}
\hline Family & Span & ID & Identity & Age & Gender & Relationship \\
\hline \multirow[t]{4}{*}{ A } & \multirow[t]{4}{*}{22 weeks } & A1 & Older adult & 80 & Male & Grandfather \\
\hline & & A2 & Middle-aged & 50 & Female & Daughter \\
\hline & & A3 & Middle-aged & 52 & Female & Daughter \\
\hline & & A4 & Young & 19 & Female & Granddaughter \\
\hline \multirow[t]{3}{*}{ B } & \multirow[t]{3}{*}{17 weeks } & B1 & Older adult & 77 & Male & Father \\
\hline & & B2 & Older adult & 75 & Female & Mother \\
\hline & & B3 & Middle-aged & 48 & Female & Daughter \\
\hline \multirow[t]{5}{*}{$\mathrm{C}$} & \multirow[t]{5}{*}{7 weeks } & $\mathrm{C} 1$ & Older adult & 81 & Male & Grandfather \\
\hline & & $\mathrm{C} 2$ & Older adult & 75 & Female & Grandmother \\
\hline & & C3 & Middle-aged & 48 & Male & Son \\
\hline & & $\mathrm{C} 4$ & Middle-aged & 45 & Male & Son \\
\hline & & C5 & Young & 24 & Male & Grandson \\
\hline \multirow[t]{3}{*}{$\mathrm{D}$} & \multirow[t]{3}{*}{4 weeks } & D1 & Older adult & 61 & Male & Father \\
\hline & & D2 & Middle-aged & 55 & Female & Mother \\
\hline & & D3 & Young & 26 & Female & Daughter \\
\hline \multirow[t]{3}{*}{$\mathrm{E}$} & \multirow[t]{3}{*}{2 weeks } & E1 & Older adult & 78 & Female & Mother \\
\hline & & E2 & Middle-aged & 52 & Female & Daughter \\
\hline & & E3 & Middle-aged & 55 & Male & Son-in-law \\
\hline \multirow[t]{3}{*}{$\mathrm{F}$} & \multirow[t]{3}{*}{4 weeks } & $\mathrm{F} 1$ & Older adult & 80 & Female & Mother \\
\hline & & $\mathrm{F} 2$ & Middle-aged & 48 & Female & Daughter-in-law \\
\hline & & F3 & Middle-aged & 53 & Male & Son \\
\hline \multirow[t]{3}{*}{ G } & \multirow[t]{3}{*}{2 weeks } & G1 & Older adult & 81 & Male & Father \\
\hline & & G2 & Older adult & 77 & Female & Mother \\
\hline & & G3 & Middle-aged & 53 & Female & Daughter \\
\hline \multirow[t]{3}{*}{$\mathrm{H}$} & \multirow[t]{3}{*}{2 weeks } & $\mathrm{H} 1$ & Older adult & 60 & Male & Father \\
\hline & & $\mathrm{H} 2$ & Middle-aged & 55 & Female & Mother \\
\hline & & H3 & Young & 27 & Female & Daughter \\
\hline
\end{tabular}

We developed and launched a WeChat mini-program in the group to lower the technical threshold for older adults to use WeRun and to facilitate our research team in collecting step data. The program is a "sub-application" within the WeChat ecosystem, enabling participants to access their chat group and the research team to access step count history. Figure 1 shows a participant view of the mini-program.

At the end of the study, we conducted 30-50 minute interviews with participants individually to understand their opinions and experiences with fitness tracking and sharing. We opted for individual interviews over group interviews to allow participants to 


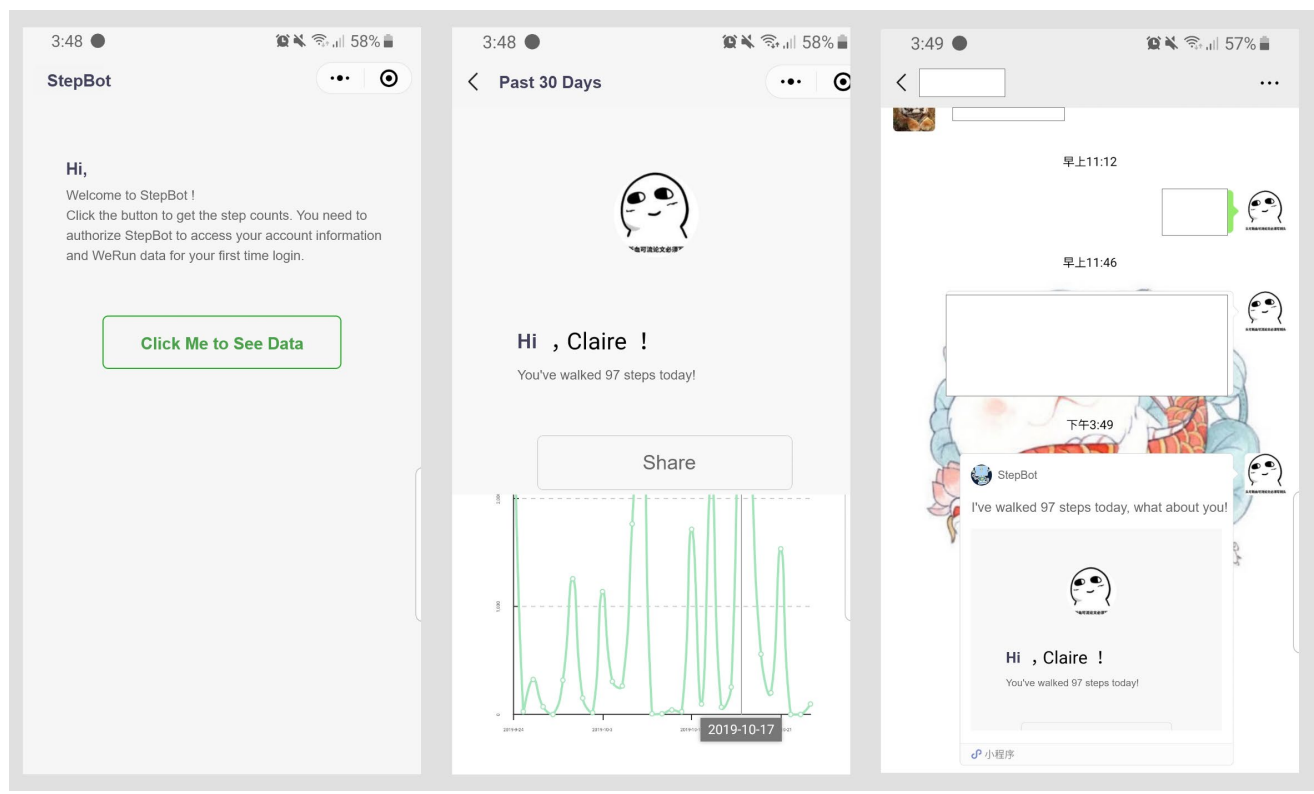

Figure 1: The interface of the WeRun mini-program.

honestly express their experiences with fitness sharing and their attitudes about family interactions.

\subsection{Data Analysis}

We report on data from the group and individual interviews and chatlog conversations collected during the study. We collected 917 minutes of interview recordings and 1198 chatlog records generated during the study period. We qualitatively analyzed the interview and chatlog data, taking an approach inspired by grounded theory [5]. We read all interview transcripts and chatlog conversations, using our initial understanding to generate a starting list of codes. We returned to the data to conduct systematic axial coding and identified the emergent themes. After several iterations of coding, we identified and categorized themes that emerged naturally, which we present in the findings. We found related quotes in the interview transcripts and translated them into English. We also tagged each message according these themes, allowing each message to be tagged with multiple themes. To compare the different engagement styles of the three age groups, we counted the number of messages in every theme sent by each participant and calculated the average number of messages in each theme sent per week per person in every age group.

Although we collected step count data from most of our participants, this paper focuses on the analysis of interview and chatlog data. All of the participants shared and discussed their daily activities with their family members in their group, but six out of 27 participants did not authorize the mini-program to access their step counts before and during the study because of technical issues and privacy concerns. These data gaps make it challenging to draw conclusions from activity outcomes, and we instead focus on discussing participant's experiences during the family tracking activity.

\section{RESULTS}

We structure the results following the three research questions. We first discuss how family tracking impacts health awareness in the family, then how sharing fitness data became a channel for family members to express care for one other, and finally the challenges of fitness sharing families faced during the study. We refer to participants with a group letter, followed by a participant number (e.g., A3, B2, C1).

\subsection{RQ1: Awareness of Each Other's Health}

We found that family members often have no previous exposure to each other's activity. Lukoff et al. [21] found that journaling nonshared experiences could extend awareness of family members' behavior and condition. In addition to journaling, we found that the shared fitness data helped participants be aware of and understand their family member's current health conditions and helped them better take care of one another.

4.1.1 Daily Steps Provide a Window into Family Members' Health and Well-Being Status. Participants found that family members' daily step counts revealed if they were well and healthy. B3, a middle-aged participant, used the step counts of her parents (B1 and B2) to observe whether they were in good condition. She said, "Since we were not visiting or calling my parents every day, 
Supporting Caring among Intergenerational Family Members by Family Fitness Tracking

it was hard for me to know my parents' condition in time. With the group, I can know their health condition through their step data and the messages they have posted. Unless on rainy or smoggy day, their step counts should always on that level." Similarly, B2 was happy to learn her family was in good health from the step data: "There are several people in our family group who can see each other's step counts. We don't need to ask each other if they're okay. Why? I'm sure they're all right when I see they've walked many steps. We don't need to worry about it." Besides health status, participants gained new understanding about their family members' activities through sharing step counts. For example, one adult child (G3) texted her parents and said, "I didn't expect that you could exercise that much, walk 20000 to 30000 [steps] per day. I thought that you were just doing your regular activities every day. Now I knew my mom was the leader in her dance group and I am proud of you."

Besides physical health, some adult children became aware of their parents' mental state by listening to voice messages they sent sharing their daily exercise. For example, a middle-aged participant (A2) used the voice messages sent by her father (A1) to understand her father's condition, "I would hear my father's voice message every day as soon as he sent. I had to listen, it's a relief when I found out that he's in a good mood and good health condition." From tracking and sharing daily steps, they also realized that their parents desired a sense of achievement and attention from their children. Most of the adult children noticed that their parents viewed exercising as extremely important. For example, in group $\mathrm{C}$, the middle-aged participant $\mathrm{C} 3$ said, "What he [C1, C3 's father] cares about is to be the first one on the leaderboard, walk more than 25000 steps every day. Sometimes when he forgot to wear the pedometer bracelet and did not record the step counts, he even wanted to take a walk again to make up for it." C3's brother C4 also commented, "[C1] valued his step counts data." They felt their parents regarded the activity as a task not only to be completed, but also to be done well.

4.1.2 Care through Data. Adult children conveyed their care by asking questions when they noticed unusual data from their parents. Both excessive exercise and unusually low step counts alerted them to pay attention to their parents' health. For example, B2 (an older participant) and B3 (B2's adult daughter) had the following conversation across in the first few days of the study.

B2: "I've taken 17191 steps today, and 26400 steps yesterday."

B3: "Why are you walking so much?"

B2: "Yes, one lap of the playground is 400 meters. I walked on the outside runway, plus the distance went [to the playground] and back [home]. I walked for over an hour."

B3: "But don't walk too much. This is only a test [family tracking study]. Healthy comes first."

[Several days later]
PervasiveHealth 20, May 18-20, 2020

Atlanta, United States, USA

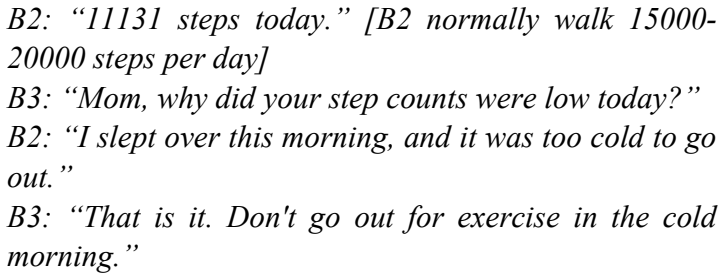

The daily steps also prompted participants to further reason about what occurred to their family members during the day. F2 once doubted the accuracy of her spouse's (F3) step counts because F3 was typically active. She (F2) said, "You've only walked around 2600 steps today. I don't really believe it." F3 then explained that, "The air outside is too dirty, so I exercised at home [without carrying the phone]." B3 was worried about her mother (B2) when seeing B2's daily step counts were only 1015 steps, which were much less than her average daily steps (15000 to 20000). Then, B3 realized that the abnormally small number of steps was because WeRun failed to acquire data from her mother's pedometer, so she asked "Mom, it seems that you posted the wrong number of steps today. Missing a digit, I think."

\subsection{RQ2: How Care is Expressed}

We found some participants inferred information about each other's health via their daily step counts, while the passive sharing of health-related step activity encouraged others to share more detailed information about their health to the group. In Yamashita et al.'s [28] study, the act of a family caregiver helping their care recipients record mundane events initiated positive health-related conversations. In our study, all generations collected their daily step counts independently. Conversations related to care were instead triggered after participants reflected on each other's data and observed unusual events, such as too many or too few daily steps, atypical schedules and experiences, and messages sent at an unusual time. We observed that the frequency of caregiving conversations stayed steady over the course of the study.

4.2.1 Conversations Triggered by the Shared Fitness Data. Besides recording and sharing daily step counts, participants also interacted and communicated with their family members around the information they shared. Participants tended to share their experiences at the same time of day, typically between $8 \mathrm{pm}$ to 11 $\mathrm{pm}$. When asked what time they usually sent messages, participants answered "it must be at night when it [WeRun] finished collecting the steps of the day (F2)" and "we're free to spend time on cell phones (F3)".

Middle-aged participants tended to lead the family interaction in the chat groups, sending 9.10 messages on average per week per person, while the older participants sent 5.83 and the younger participants sent 6.13 messages per week per person. As shown in Figure 2, family conversations not only centered on physical exercise, but also their daily activity, health care and well-being, 
encouragement and motivation, and technical difficulties of using fitness trackers.

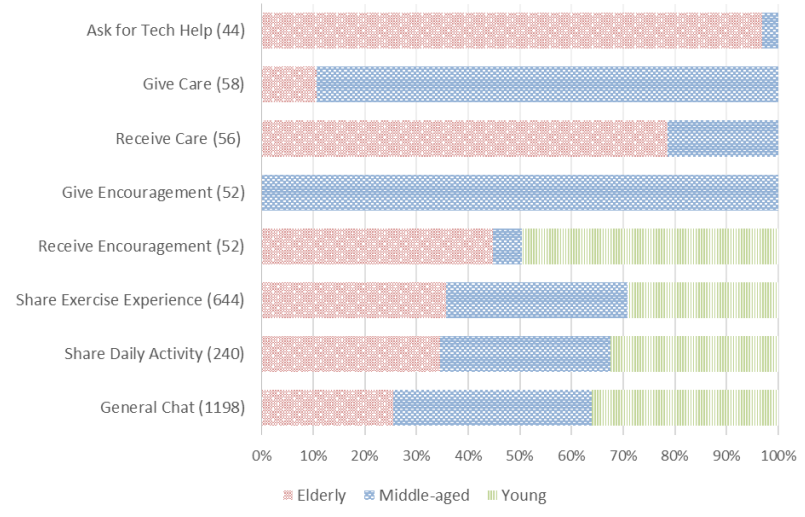

Figure 2: The percentage of the themes of messages sent by three age groups to their family tracking groups per week per person.

During the interviews, participants felt that the family tracking activity enhanced their communication by providing a platform on which they could share their feelings and thoughts with their families. Before participating in the study, all of the participating families had a family chat group. However, most of them only sent messages to that group on rare occasions such as to ask for help, to share travel pictures, or to share funny things about their children. In these groups, members often did not know what to say if they had nothing important to communicate. They were also afraid to disturb others if they sent too many messages, as E2 said, "There are more than a dozen people in our original family WeChat group. If the matter involves a small number of people, we will discuss it in private, so as not to disturb a large group of other people... In this "sports group", we talk about exercise and what everyone has done every day mainly." However, in the tracking-themed chat group, participants felt like the shared topic of daily step counts and physical exercise served as an icebreaker that could lead to other conversations. For example, when an older adult couple (G1 and G2) attempted to explain to their daughter (G3) their exercise routines, they also shared other health information related to exercise as well:

G1: "Impressive! You [G3] walked a lot. We did our routine workout. And this afternoon we went to get a new pair of eyeglasses. But there's some issue with the fundus examination."

G3: “@G1 What's wrong with the fundus, does it serous?”

G1: "I did two sessions of Taichi followed the tutorial on computer and felt really good. I have arteriosclerosis and my fundus was not clear to be seen. But it's no big deal, my vision is 0.7."
Like this chatlog, our analysis shows that family members encouraged each other by sending text messages, stickers, and thumbs-up when they achieved their exercise goals and had high step counts. Interestingly, most encouragement messages shown in the chat logs were sent by the middle-aged participants. They often paid close attention to the fitness shared by others, asked why their family members walked less, and then encouraged them to continue exercising if they could. For example, an adult child (F3) noticed that his mother (F1) stayed at home more often lately, so he encouraged her to go out more often to visit friends and visit scenic spots, "Grandma [F1] used to stay at home all the time when she had nothing to do. Now, my wife [F2] and I encouraged her to go out and hang out more with her friends as exercising."

Although the middle-aged generation often gave care to other family members, they were not always in the role of caregiver, and older adults were not always care receivers. For example, in group E, the older participant (E1), who lived geographically separated from her daughter, often expressed care about her daughter (E2) and reminded E2 to sleep on time when she noticed her daughter was replying to messages at midnight.

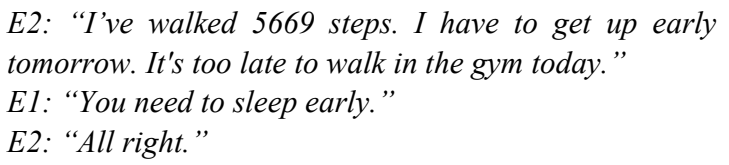

4.2.2 In-Person Care Prompted by Fitness Sharing. We found that family tracking activities not only enhanced online communications and interactions, but also promoted offline interactions between family members. Our findings parallel Lukoff et al. [21], who found tangible support occurred among family members journaling and sharing their eating behaviors.

Some participants expressed their desire to interact in-person with their family members during the study. One older adult participant (A1) expressed to his children that he would like to invite them to visit him and have dinner together, "I went out for a walk after two o'clock and had a haircut, at least [I walked] over 5000 [steps]... I felt OK. It just I will be alone this evening, [I am] waiting to see if anyone will come back to have dinner with me." His daughter A2 immediately replied to her father, "I'm going back to have dinner with Dad. $\wedge^{\wedge} \wedge$ "

Difficulties in the use of the tracking technology also led to tangible support, extending Lukoff et al.'s [21] findings where all tangible support were triggered by tracking records. Since many older adult participants in the study were new to WeChat and WeRun, sharing fitness data created opportunities for the younger family members to visit their parents to teach them how to use WeRun and WeChat. Most of the family groups encountered a situation where the older adult participant was not familiar with a feature of WeChat or WeRun, and it was difficult for other family members to explain and demonstrate the feature through WeChat or a phone call. For example, B3 visited her parents (B1 and B2) more frequently than before to help them update step data from the 
Supporting Caring among Intergenerational Family Members by Family Fitness Tracking

pedometer to WeRun. B3 recalled in her final interview, "One day, my dad's [B1] pedometer didn't work well, he immediately asked me when would I go visit them. Dad [B1] asked me, 'why my bracelet is not in sync [with WeRun]. Look at how many step counts I have in the bracelet, but how less on WeChat.' Anyway, because older adults are a bit like children, he always says, 'I have to show my number of steps, I don't care, I just want to have this. " As these quotes show, family sharing also turned into in-person visits and more time spent together in our study.

\subsection{RQ3: Challenges Family Members Face}

Tracking fitness data in a family setting provided multiple motivations for sharing health information and opportunities for caring among family members. Nevertheless, some participants were also concerned about the intervention interfering with their privacy and independence. They also worried that the act of tracking might motivate them to exercise beyond an appropriate level.

4.3.1 Tracking Step Counts is Limited and One-Sided. Although participants found daily step counts useful in providing a window into their and each other's activity, many gradually realized that daily steps were not enough to reflect physical exercise. Focusing on steps led them to pay more attention to the numbers rather than the actual experience of their family members, aiming to maximize the number of steps instead of enjoying physical exercise or maintaining health. A young participant (D3) recalled in her final interview, "Before using WeRun [to track and share step counts with family members], I sometimes thought about going out and jogging two laps at night. After jogging, I might share my experience with them. It could be some feelings after exercising or anything else. Especially after jogging outside, I would see some new scenery, or see something interesting that I wanted to share with my parents. But after using WeRun [to track and share step counts with family members], I might only focus on the total number of steps, not on the experience itself."

Participants also expressed concerns about avoiding sports injuries or over-exercising. For example, one older participant (H1) who underwent a leg surgery a year before said, "I would receive a lot of encouragement and feel happy and a sense of achievement when I reached 10000 steps. But in the meantime, I also worried if I over-exercised, which was bad for my leg."

4.3.2 Unfamiliarity of technologies in older generation. Participating in the family groups encouraged older adults to learn and practice using WeChat. A middle-aged participant (G3) mentioned that she and her parents talked more than before because her parents became more proactive in sharing and communicating in the WeChat group after they learned to use the messaging functions, "I taught them how to use WeChat before, but they often forgot. In these two weeks, they practiced every day to send text and voice messages in the group; now they fully mastered the chat functions in WeChat."
PervasiveHealth 20, May 18-20, 2020

Atlanta, United States, USA

In the initial interviews, we found that all of the middle-aged and young participants contacted their family members regularly through WeChat. They would only communicate through phone or in person only if they had something important or urgent to discuss. By contrast, although most of older adults had sufficiently learned how to use WeChat and WeRun after a few days in the study, some of them were still not comfortable with the technology. During the study, an older participant (C1) struggled to use WeChat. He therefore preferred to read others' messages and listen to what others said, but was reluctant to share his own experience and feelings, "I posted the steps counts online every day. I only like to read [other's text] and listen [to other's voice messages], don't like to send [messages], don't like to send things. Because I'm a little slow, I don't want to send things."

In addition, it was also a challenge for some of older adults to track their fitness data using WeRun. WeRun can import fitness data from compatible fitness tracking applications and automatically share real-time fitness data with family members. However, some participants, especially older adults, preferred to wear a pedometer band instead of carrying a mobile phone with them. They then had to pair their pedometer band to their mobile phone by Bluetooth to update the records to WeRun. As an older participant B2 reported, "I don't like to bring my cell phone with me. Many times, when I check WeRun at night, I found my pedometer didn't connect with my phone. I am very anxious, but sometimes it connect by itself later. I don't know why." Although users bear little responsibility in acquiring, processing, visualizing, and sharing the collected step counts, it was still a challenge for older adults to update their records from pedometers to WeRun. Older adults would send messages in the group to ask for help when they found that their WeRun records were far less than they had anticipated and did not know how to fix it.

4.3.3 Privacy Concerns and Deferred Time Commitment. Younger participants were reluctant to engage with the app and the study, worrying that tracking as a family would invade their independence and privacy. C5, a graduate student, felt uncomfortable when his family observed his step count every day and compared his data with others, "They thought that my grandpa is 80 years old and still takes 30,000 steps a day, and I am so young but walk so little every day, and I feel very embarrassed to be educated by my grandfather as he always reminds me that I am not exercising enough." A4, a college student, felt stressed and supervised by her father because he would always "like" her on the step leaderboard. She thought the "like" meant "I know what you have done and where you have been these days." A4 said, "But my father and I haven't communicated in WeChat for a long time, and we haven't talked about exercise. I sometimes suddenly saw a long list of his 'likes' to my step counts. He just 'liked' me every day. I wondered if he just wanted to get my attention, or if he deliberately found me in his contact list of three or five hundreds people, and gave me a 'like'. It made me feel as if I was being watched." Due to their busy schedules, younger participants also struggled to 
"spare a whole period of time (A4)" to read all chat records and keep up with the topics their family members discussed. A4 said it is difficult for her to participate because she received many voice messages every day from her family group, and she could only randomly pick a few voice messages to listen. "I didn't know if they said something individually or they had a specific topic, and then everyone started talking. If I was going to understand the topic, I had to listen to every voice message, which was very timeconsuming. Most of the time I was in a classroom or library."

\section{DISCUSSION}

Our results suggest that family tracking can enhance health awareness in the family by providing information about each other's health via their daily step counts. Sharing fitness data became a channel for family members to express care for one other when they observed unusual practices such as too many or too few daily steps, an abnormal schedule or experience, or messages sent at an atypical time. Nevertheless, sharing fitness data among family members led to privacy, independence, and potential health issues among participants. Our results show two unique aspects of family fitness tracking and sharing: (1) family sharing differs from social network sharing, and (2) generations have different expectations and preferences with fitness tracking.

\subsection{Family Sharing is Different from Social Network Sharing}

Contrasting with previous studies suggesting accuracy and step counts are less important in social network fitness sharing [11], our participants paid close attention to the total numbers and the accuracy of step counts. In a family context, the accuracy of tracked data might be essential because it reflects not only individuals' own condition, but surfaces their progress to others who are using the data to judge their health status. Family members reminded, cared for, and encouraged each other based on their reflection on the shared data. They expected the recorded step counts to be generally accurate.

Some of our participants desired a description of how WeRun collected and processed their data because sometimes they found that the results shown by WeRun did not match their expectations. Yang et al. [30] similarly found users of personal tracking technologies were uncertain about the accuracy of their devices, suggesting that designs could support testability, allow greater enduser calibration, and increase transparency [30]. In our study, participants offered their own interpretations when they believed their physical exercise cannot be measured by the system. Our findings suggest that it can be helpful to provide mechanism to allow people to reflect on the accuracy of their tracked data. If the result varies from how much they think they exercised, designs could prompt them to think whether they forgot to carry the phone or whether they were exercising in forms which the device could not capture. Such a mechanism could help users to alleviate doubts and be better prepared to address unexpected results.

When sharing tracked data on social networking sites, Gui et al.'s participants were aware that their contacts had diverse ages, occupations, health statuses, and lifestyles, and considered the individual situations when interpreting and comparing contacts' step counts [11]. By contrast, some of our participants placed substantial value on the data and regularly used it to compare family members. However, family members also worried about potential health issues resulting from becoming motivated to exercise beyond an appropriate level. In previous studies [16], researchers found that providing uncertainty when presenting information could help participants make better decisions and alleviate anxiety when the demonstrated information did not match their expectation. When showing tracked fitness data in a family tracking system, instead of using an exact number, one could use a visualization which shows if a person's daily step count falls within the range of the person's normal daily step counts. In addition to helping users move away from solely maximizing step counts, systems could prompt users to recall their activity experiences when reflecting on their data. In our study, some participants shared their bodily feelings such as being tired, sore, and lazy, when they reported daily step counts. Tracking systems could collect this feedback to predict and share these feelings based on the person's step count of the day.

\subsection{Designing for the Intergenerational Family}

In our study, family members were given equal roles in their chat group. However, our findings suggest the three age groups displayed distinctly different behaviors and it may be insufficient to design family tracking technology the same for everyone.

Older adults attached great importance to the group channel, caring highly about how others saw their activities. Although some older adult participants struggled to learn to use technology, once they had mastered it, they appreciated being able to better communicate with their children. They treated the family tracking activity seriously. They regarded it as a task not only to be completed but also to be done well, and many felt disappointed if their step counts were not recorded due to technical issues. Therefore, when designing fitness tracking applications for older adults, designers need to help them gain a sense of achievement, reduce the complexity of procedures, guide them to learn and practice the features, and provide a convenient platform for them to ask for help from their families.

Rewards systems can motivate older adults and help them gain a sense of achievement. For example, Herpich and colleagues conducted a workshop to co-design an encouragement system for older adults [12], finding that participants felt rewarded when the activity itself or its outcome positively influenced them. In our study, the older participants felt rewarded when their family members provided praise, gave "thumb ups", and was influenced 
Supporting Caring among Intergenerational Family Members by Family Fitness Tracking

by them to exercise. Tracking applications could guide family members to encourage older adults by providing badges, stickers or achievement stamps which could be sent between users. Previous studies found many older adults consider existing social media lightweight, in contrast to their desire to engage in thoughtful communication that often requires an investment of time and attention to composition $[13,19,20]$. Designers could incorporate features which encourage and support adult children in conducting deep and thoughtful communications with older adults like conversation prompts.

It is also important to reduce the complexity of apps and make procedures as automated as possible from collecting and reflecting to sharing and discussing. In our study, older adults struggled to pair their pedometer band to their mobile phone by Bluetooth to update the records to WeRun. Designers could use onboarding systems or daily tasks to guide older adults to learn and practice these features.

Middle-aged participants often served as the main caregiver and supporter to both the younger and older generations. They typically took the lead role in caring for others, paying attention not only on their own data but to other family members' data to notice differences and reason through what happened. They listened to voice messages to pick up any subtle cues which could imply the physical and mental condition of their family members.

The design of family fitness tracking technologies should lower their burden to reflecting on tracked data. Our results suggest that this generation is primarily searching for data which deviates from their family members' daily routines to keep aware of others' situations. It could be helpful if a tracking tool could identify unusual data caused by internal factors (e.g., the health or mood of participants) and unusual data caused by external factors (e.g., weather and technical issues). In this study, unusual data, such as too many or too few steps, triggered interactions between family members. Most of the time, unusual data was caused by external factors. But occasionally, it means that the producer of the data might be in a different condition than usual, and thus might need help. It could also help this generation if tools could send reminders to users when detecting abnormal conditions.

Young participants paid more attention to their independence and privacy than their parents and grandparents who attached significant importance to the collective value of tracking as a family. They were also more familiar with recent technologies and wanted to experiment with different products. Younger participants also had difficulties engaging in the conversations due to their fragmented attention and lower commitment to engaging. It would be helpful to protect privacy and independence by allowing users to customize what data they shared rather than presume that all participants share information equally. Automatically transcribing voice messages could additionally make it easier for younger generations to quickly scan the ongoing conversation and engage with it.

The generations in our study had different expectations and preferences with fitness tracking and social practices, and it can be
PervasiveHealth 20, May 18-20, 2020

Atlanta, United States, USA

challenging to support all of them. However, we suggest designs support different levels of engagement and expertise for different generations. When users are not familiar with the more complex functions, it does not affect their use of simple basic functions such as tracking and sharing. As they become more skilled and comfortable with the tools, users can further explore more complex features, such as customizing a digital profile and using a reward system to send stickers to other family members.

\section{LIMITATIONS AND FUTURE WORK}

We collected data from three different age groups; however, younger participants were somewhat underrepresented in our study with only four participants. We suspect that other younger adults may have perspectives and opinions that we were not able to report on. To better understand the challenges this generation faces in tracking as a family, future work could sample this group more heavily. In addition, because of their privacy concerns, we were not able to collect all participants' daily step counts. Future work could investigate how family-based tracking interventions could influence the exercise level of each generation. In future work, it would be beneficial to understand the influences of different living arrangements (e.g., by comparing families living together versus separately), and to consider families with varied socioeconomic statuses. The ubiquity of WeChat and WeRun made them appropriate tools for understanding and supporting intergenerational activity tracking in China. Future work can complement this by considering perspectives from other cultures.

\section{CONCLUSION}

Tracking in a family context has the potential to increase social support and help families build awareness, reflect on health behaviors, and set goals. We explored how a physical activity intervention can help support care among intergenerational family members, finding that family tracking triggered different levels of information sharing and different forms of caring. Older adults, middle-aged, and young participants displayed distinctly different use and care behaviors, making it challenging to design family tracking technologies the same for everyone. Family sharing is additionally distinct from social network sharing, requiring higher accuracy and explanation for how data is collected and analyzed. Finally, we suggest that tracking tools account for varied levels of expertise and engagement to account for the preferences and practices of each generation.

\section{ACKNOWLEDGMENTS}

This work was funded in part by the National Science Foundation under award IIS-1850389. We thank Qiancheng Wu for his contribution to the development of the WeChat mini-program and Matthew Bietz for feedback on the project. 


\section{REFERENCES}

[1] Jomara Binda, Chien Wen Yuan, Natalie Cope, Hyehyun Park, Eun Kyoung Choe, and John M. Carroll. 2018. Supporting effective sharing of health information among intergenerational family members. In ACM International Conference Proceeding Series. DOI:https://doi.org/10.1145/3240925.3240936

[2] Clara Caldeira, Matthew Bietz, Marisol Vidauri, and Yunan Chen. 2017. Senior care for aging in place: Balancing assistance and independence. In ACM Conference on Computer Supported Cooperative Work, CSCW. DOI:https://doi.org/10.1145/2998181.2998206

[3] Nathalie Colineau, Dipak Bhandari, Cécile Paris, Yanfeng Shu, and Peter Marendy. 2009. Supporting family engagement in weight management. In Conference on Human Factors in Computing Systems. DOI:https://doi.org/10.1145/1520340.1520606

[4] Sunny Consolvo, Katherine Everitt, Ian Smith, and James A. Landay. 2006. Design requirements for technologies that encourage physical activity. In Conference on Human Factors in Computing Systems. DOI:https://doi.org/10.1145/1124772.1124840

[5] Juliet Corbin and Anselm Strauss. 2012. Basics of Qualitative Research (3rd ed.): Techniques and Procedures for Developing Grounded Theory. DOI:https://doi.org/10.4135/9781452230153

[6] Daniel A. Epstein, Bradley H. Jacobson, Elizabeth Bales, David W. McDonald, and Sean A. Munson. 2015. From "nobody cares" to "way to go!": A design framework for social sharing in personal informatics. In the 2015 ACM International Conference on Computer-Supported Cooperative Work and Social Computing. DOI:https://doi.org/10.1145/2675133.2675135

[7] Lizbeth Escobedo, Raina Ahuja, Elmer Barrera, Connie Guan, Enrique Zavala Robert El-Kareh, Jihoon Kim, Kyung E. Rhee, Wei Peng, Guoliang Xing, Chuongguang Bi, and Jina Huh. 2017. SHINE-L: Sensing health and family behavior routines for Latino families. In Conference on Human Factors in Computing Systems. DOI:https://doi.org/10.1145/3027063.3053231

[8] Qiushi Feng, Wei Jun Jean Yeung, Zhenglian Wang, and Yi Zeng. 2019. Age of Retirement and Human Capital in an Aging China, 2015-2050. Eur. J. Popul. (2019). DOI:https://doi.org/10.1007/s10680-018-9467-3

[9] Hasan Shahid Ferdous, Bernd Ploderer, Hilary Davis, Frank Vetere, Kenton O’Hara, Geremy Farr-Wharton, and Rob Comber. 2016. TableTalk: Integrating personal devices and content for commensal experiences at the family dinner table. In the 2016 ACM International Joint Conference on Pervasive and Ubiquitous Computing. DOI:https://doi.org/10.1145/2971648.2971715

[10] Andrea Grimes, Desney Tan, and Dan Morris. 2009. Toward technologies that support family reflections on health. In the 2009 ACM SIGCHI International Conference on Supporting Group Work DOI:https://doi.org/10.1145/1531674.1531721

[11] Xinning Gui, Yu Chen, Clara Caldeira, Dan Xiao, and Yunan Chen. 2017. When fitness meets social networks: Investigating fitness tracking and social practices on WeRun. In Conference on Human Factors in Computing Systems. DOI:https://doi.org/10.1145/3025453.3025654

[12] Madita Herpich, Thomas Rist, and Elisabeth André. 2017. Co-designing a recommender system for the elderly. In ACM International Conference Proceeding Series. DOI:https://doi.org/10.1145/3056540.3064948

[13] Alexis Hope, Ted Schwaba, and Anne Marie Piper. 2014. Understanding digital and material social communications for older adults. In Conference on Human Factors in Computing Systems. DOI:https://doi.org/10.1145/2556288.2557133

[14] Kori Inkpen, Brett Taylor, Sasa Junuzovic, John Tang, and Gina Venolia. 2013 Experiences2Go: Sharing kids' activities outside the home with remote family members. In the ACM Conference on Computer Supported Cooperative Work, CSCW. DOI:https://doi.org/10.1145/2441776.2441926

[15] Tejinder K. Judge, Carman Neustaedter, Steve Harrison, and Andrew Blose. 2011. Family Portals: Connecting families through a multifamily media space. In Conference on Human Factors in Computing Systems. DOI:https://doi.org/10.1145/1978942.1979122
[16] Matthew Kay, Tara Kola, Jessica R. Hullman, and Sean A. Munson. 2016. When (ish) is my bus? User-centered visualizations of uncertainty in everyday, mobile predictive systems. In Conference on Human Factors in Computing Systems. DOI:https://doi.org/10.1145/2858036.2858558

[17] Elizabeth Kaziunas, Mark S. Ackerman, Silvia Lindtner, and Joyce M. Lee. 2017. Caring through data: Attending to the social and emotional experiences of health datafication. In the ACM Conference on Computer Supported Cooperative Work, CSCW. DOI:https://doi.org/10.1145/2998181.2998303

[18] Ian Li, Anind Dey, and Jodi Forlizzi. 2010. A stage-based model of personal informatics systems. In Conference on Human Factors in Computing Systems. DOI:https://doi.org/10.1145/1753326.1753409

[19] Siân E. Lindley. 2012. Shades of lightweight: Supporting cross-generational communication through home messaging. Univers. Access Inf. Soc. (2012) DOI:https://doi.org/10.1007/s10209-011-0231-2

[20] Sian E. Lindley, Richard Harper, and Abigail Sellen. 2009. Desiring to be in touch in a changing communications landscape: Attitudes of older adults. In Conference on Human Factors in Computing Systems. DOI:https://doi.org/10.1145/1518701.1518962

[21] Kai Lukoff, Taoxi Li, Yuan Zhuang, and Brian Y. Lim. 2018. TableChat: Mobile food journaling to facilitate family support for healthy eating. Proc. ACM Human-Computer Interact. (2018). DOI:https://doi.org/10.1145/3274383

[22] E. D. Mynatt, J. Rowan, A. Jacobs, and S. Craighill. 2001. Digital family portraits: Supporting peace of mind for extended family members. In Conference on Human Factors in Computing Systems.

[23] Carman Neustaedter, Kathryn Elliot, and Saul Greenberg. 2006. Interpersonal awareness in the domestic realm. In ACM International Conference Proceeding Series. DOI:https://doi.org/10.1145/1228175.1228182

[24] Laura R. Pina, Sang Wha Sien, Teresa Ward, Jason C. Yip, Sean A. Munson, James Fogarty, and Julie A. Kientz. 2017. From personal informatics to family informatics: Understanding family practices around health monitoring. In the ACM Conference on Computer Supported Cooperative Work, CSCW. DOI:https://doi.org/10.1145/2998181.2998362

[25] Natalia Romero, Panos Markopoulos, Joy Baren, Boris Ruyter, Wijnand IJsselsteijn, and Babak Farshchian. 2007. Connecting the family with awareness systems. In Personal and Ubiquitous Computing. DOI:https://doi.org/10.1007/s00779-006-0089-0

[26] Herman Saksono, Ashwini Ranade, Geeta Kamarthi, Carmen Castaneda-Sceppa Jessica A. Hoffman, Cathy Wirth, and Andrea G. Parker. 2015. Spaceship launch Designing a collaborative exergame for families. In the 2015 ACM International Conference on Computer-Supported Cooperative Work and Social Computing. DOI:https://doi.org/10.1145/2675133.2675159

[27] Chris Schaefbauer, Danish Kahn, Amy Le, Garrett Sczechowski, and Katie Siek. 2015. Snack buddy: Supporting healthy snacking in low socioeconomic status families. In the 2015 ACM International Conference on Computer-Supported Cooperative Work and Social Computing. DOI:https://doi.org/10.1145/2675133.2675180

[28] Naomi Yamashita, Hideaki Kuzuoka, Keiji Hirata, Takashi Kudo, Eiji Aramaki, and Kazuki Hattori. 2017. Changing moods: How manual tracking by family caregivers improves caring and family communication. In Conference on Human Factors in Computing Systems. DOI:https://doi.org/10.1145/3025453.3025843

[29] Naomi Yamashita, Hideaki Kuzuoka, Takashi Kudo, Keiji Hirata, Eiji Aramaki, and Kazuki Hattori. 2018. How information sharing about care recipients by family caregivers impacts family communication. In Conference on Human Factors in Computing Systems. DOI:https://doi.org/10.1145/3173574.3173796

[30] Rayoung Yang, Eunice Shin, Mark W. Newman, and Mark S. Ackerman. 2015. When fitness trackers don't "fit": End-user difficulties in the assessment of personal tracking device accuracy. In the 2015 ACM International Joint Conference on Pervasive and Ubiquitous Computing. DOI:https://doi.org/10.1145/2750858.2804269 